\title{
Differential GFR estimation of the double moiety kidneys by modified GATE'S formula
}

\author{
Sivasubramaniyan. $\mathrm{V}^{1,2}{ }^{*}$, Sai chaithanya Teja. $\mathrm{M}^{3}$ and venkataramaniah. $\mathrm{K}^{3}$ \\ ${ }^{\mathbf{1}}$ Doctoral Research Scholar, Department of Physics, Sri Sathya Sai Institute of Higher Learning, Prashanthi Nilayam, Puttaparthi, Anantapur, \\ Andhra Pradesh, India. \\ ${ }^{2}$ Professor of Nuclear Medicine, Department of Nuclear Medicine, PETCT \& Molecular Imaging, Sri Aurobindo Institute of Medical Sciences, Indore, \\ Madyapradesh. \\ ${ }^{3}$ Department of Physics, Sri Sathya Sai Institute of Higher Learning, Prashanthi Nilayam, Puttaparthi, Anantapur, Andhra Pradesh, India.
}

*Correspondence Author: Sivasubramaniyan, Professor of Nuclear Medicine, Department of Nuclear Medicine, PETCT \& Molecular Imaging, Sri Aurobindo Institute of Medical Sciences, Indore, Madyapradesh.

Received date: February 17, 2021; Accepted date: March 08, 2021; Published date: March 20,2021

Citation: Sivasubramaniyan, chaithanya S. and venkataramaniah (2021) Differential GFR estimation of the double moiety kidneys by modified GATE'S formula. Clinical Medical Reviews and Reports. 3(3); DOI: 10.31579/2690-8794/067

Copyright: (C2021 Sivasubramaniyan, This is an open access article distributed under the Creative Commons Attribution License, which permits unrestricted use, distribution, and reproduction in any medium, provided the original work is properly cited.

\section{Abstract}

Aim: This retrospective analysis of the GFR values of the individual moieties obtained by using a novel software with modified GATE'S formula in the in house software created at SSSIHL, compared and evaluated with the value derived by the combined GFR value of the single kidney GFR as obtained from the Icon software loaded in the gamma camera.

Materials and Method: This retrospective study of 10patients diagnosed with unilateral double moiety and 5 patients with bilateral duplication was undertaken to validate the modified GATE'S formula in house software created at SSSIHL. This group had 12 males in the age range of 03-66 years with the mean age of $26.5 \pm 2$ years and 3 females in the age range of $29-31$ years with mean age of $31 \pm 2$ years. Renal scans were done using Siemens dual head gamma camera (E.CAM). The radiopharmaceutical 99mTc-DTPA $5 \mathrm{mCi}$ was injected as a bolus intravenously for the adult patients and $3 \mathrm{mCi}$ was injected for the children. The images were obtained and processed by using GFR/Renal differential processing protocol in icon software. Later the raw data of the scan study transferred to the workstation loaded with the in house software created at SSSIHL with modified GATE'S formula created by us. The individual moiety GFR values were calculated using the software with modified Gate's formula developed in MATLAB GUI algorithm and tabulated. Then the combined value of individual moiety GFR values compared with that of the individual whole kidney GFR value obtained by the Icon software protocol.

Result: The T test evaluation done in the online statistical calculator of Maths Portal org revealed that the mean values of the two groups showed no significant difference at $\mathrm{p}<0.05$. The calculated $\mathrm{t}$ value is smaller than the critical value $(0.0217<2.024)$, so the means are not significantly different. Pearson Correlation performed between the above two samples using statistical online calculators also showed the correlation coefficient of $r=0.9247$ and a mean difference of 0.09 indicating that the high correlation between them. The observed significance $\mathrm{p}$ value $<0.0001$ at $\mathrm{t}$ value of 10.27 , showed no significant difference detected between the two samples.

Conclusion: It can be concluded that the in house software created at SSSIHL with modified GATE'S formula method of GFR calculating software showed identical values with that of the GFR calculated with the original Gate's formula. This was found to be useful in calculating the GFR of individual moieties separately in the cases of Double moieties in both Unilateral and Bilateral involvement effectively. The utility and usefulness of the separate estimation of individual moieties GFR needed for Nephron Sparing Surgery.

Key words: 99m Tc-DTPA renography, gamma camera, double moiety kidneys, modified GATE'S formula in the in house software created at SSSIHL

\section{Introduction}

The double moiety of the kidney is a congenital anomaly in which the usual single renal collecting system is replaced with two separate moieties with separate ureters with the absence of renal pelvis. This can be either Unilateral or bilateral afflictions. The abnormal moiety is usually associated with ectopic ureters accompanied by vesico-ureteric reflux resulting in impaired functioning of the moiety. For the decision of partial nephrectomy which is preferred now than the previously utilized heminephrectomy, the differential GFR values of the individual moieties is a must. The good and adequate functional capacity of the one of the moieties is essential to go for the Nephron sparing surgery. 
The theory and practice of quantitative renography with I 131 Hippuran was introduced by Van Stekelen burg et.al [1].The utility and usefulness of the dynamic Tc $99 \mathrm{~m}$ DTPA renal scintigraphy for the assessment of glomerular filtration rate was reported by Klopper JF [2]. Houston et.al introduced the four compartmental model analysis and calculation for In 111 DTPA and Tc 99m DTPA renographies [3]. Later it was developed as the Gates formula by Gory F.Gateset.al whcih had been put into use in almost all commercially available software packages [4, 5and 6]. The nephrology text books and the nephrologist talk about the GFR estimation of the individual moieties, till today there is no commercial software available in the market or in the web resources for drawing the two moieties in a single kidney outline and proceed with the calculation. Probably the upper and lower moieties of the single kidney only are drawn and GFR calculation is made using the available software. This is scientifically not acceptable. The situation is further complicated in the case of bilateral duplication of moieties. Hence the need for the modification of the Gate's formula with the provision to split the kidney outlines is a must in those with renal duplication. Then only the correct GFR value of the individual moieties can be estimated confidently.

In this scenario we developed in house software created at SSSIHL for the evaluation of individual moiety GFR using the Math lab algorithm and graphical user interface to draw and calculate the individual GFR of the moieties introducing the six compartmental model just by modifying the regular Gates formula based on the four compartmental model [7]. The renal software utilizes gate's formula for the calculation of the GFR. The parameters like height of the patient (in $\mathrm{cm}$ ), weight (in $\mathrm{Kg}$ ) and the age (in years) are given as input according to the program.

$$
\text { Total renal uptake }(\%)=
$$

$$
\frac{\left[\frac{R-R B}{e^{-\mu x}}+\frac{L-L B}{e^{-\mu x}}\right]}{\text { (Preinjection - Postinjection counts) }}
$$

where, $\mathrm{R}=$ right kidney counts, $\mathrm{RB}=$ right kidney background counts, $\mathrm{L}$ $=$ left kidney counts, $\mathrm{LB}=$ left kidney background counts and $\mu=$ linear attenuation co-efficient of $99 \mathrm{mTc}$ in soft tissue which is equal to 0.153 and $\mathrm{x}$ is kidney depth given by the equations below which are also known as Tonnesen equations. Depth of the kidney is derived by multiplying the $13.2 \mathrm{x} \mathrm{y}+0.7$. Where $\mathrm{y}$ is the ratio of Weight in $\mathrm{kg}$ divided by Height in $\mathrm{cm}$ of the patient. The total GFR is calculated form the renal uptake RU $\mathrm{X}(9.8127-6.82519$. Similarly the individual GFR values of the kidneys are derived from the RU value as follows.

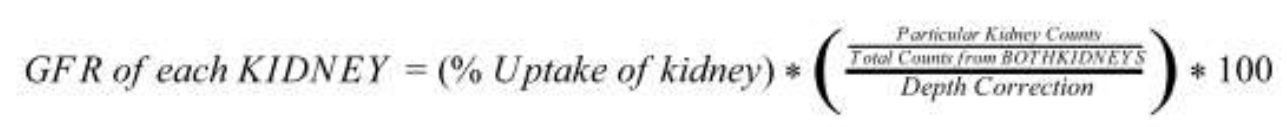

In our in house software created at SSSIHL with modified Gates formula the calculation of total kidney uptake RU value is similar to that of the original formula. After wards it splits the individual kidney GFR calculation into individual upper moiety and lower moiety fractions as shown below based on the mathematically proven six compartmental model analysis.

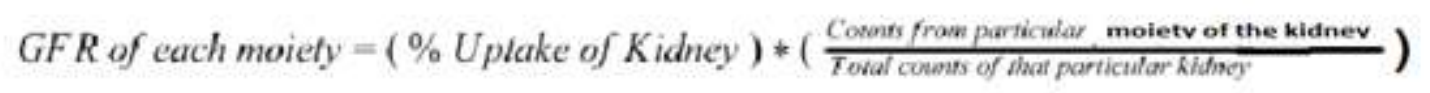

The GUI interface with the facility of drawing the double moieties in case there is renal duplication or choosing the single kidney option is shown below in the Figure 1.

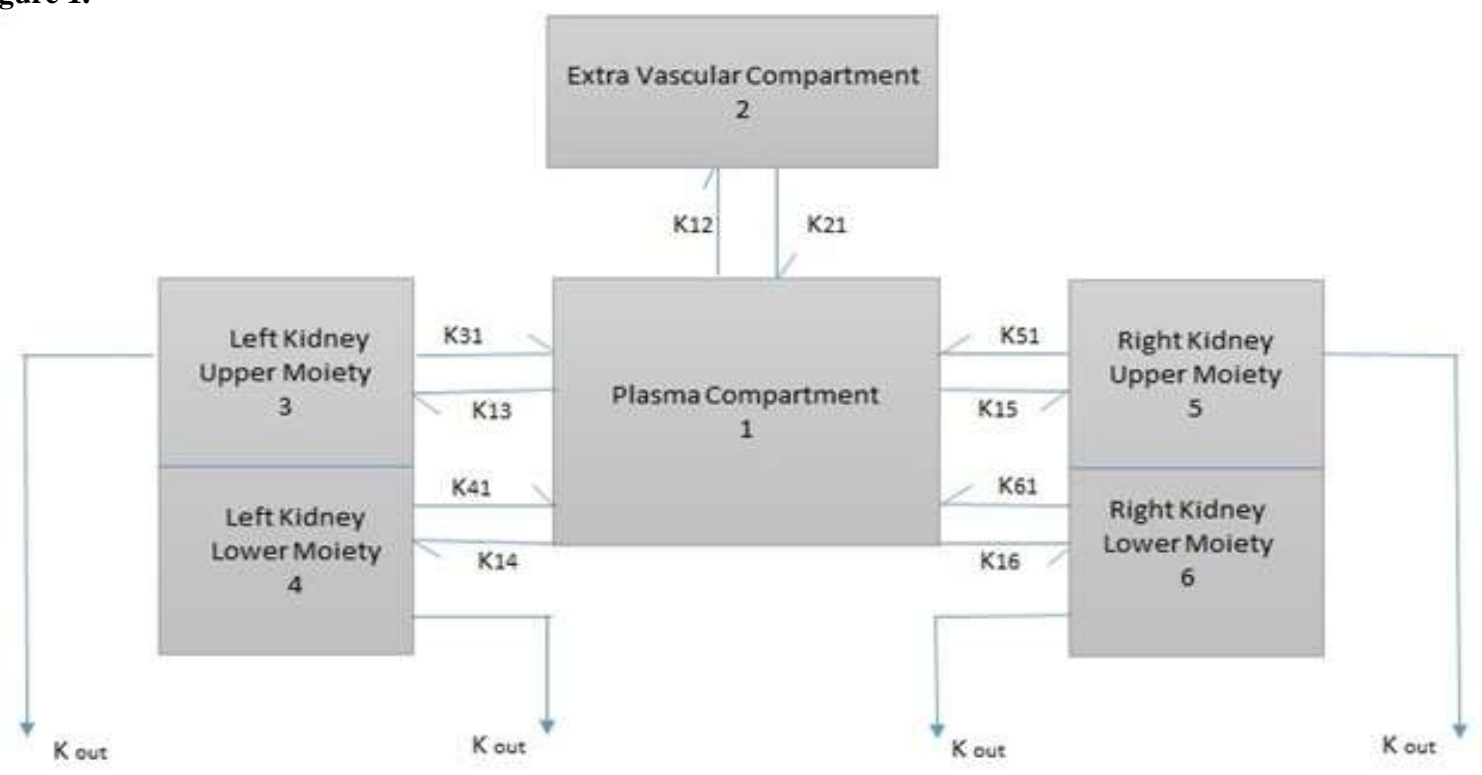


The method of drawing and selecting the individual moieties in the case of a bilateral double moiety is shown in Figure 2.

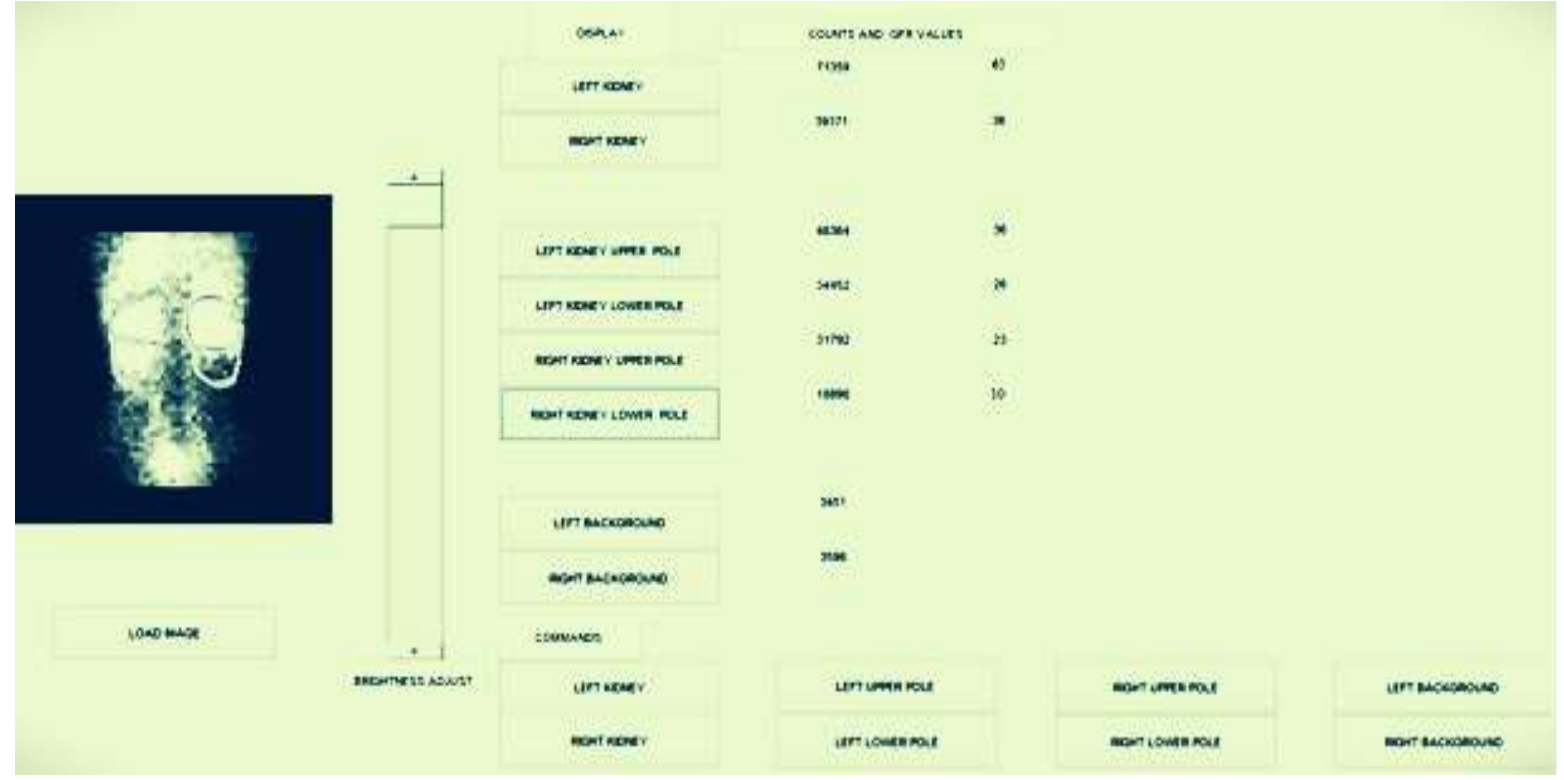

\section{Materials and Methods}

Renal scans were done using Siemens dual head gamma camera (E.CAM). The radiopharmaceutical 99mTc-DTPA $5 \mathrm{mCi}$ was injected as a bolus intravenously for the adult patients and $3 \mathrm{mCi}$ was injected for the children. The images were obtained and processed by using GFR/Renal differential processing protocol in icon software. Later the raw data of the scan study transferred to the workstation loaded with the in house software created at SSSIHL with modified GATE'S formula created by us. The individual moiety GFR values were calculated using in house software created at SSSIHL with modified Gate's formula developed in MATLAB GUI algorithmand tabulated. Then the combined value of individual moiety GFR values compared with that of the individual kidney GFR value obtained by the Icon software protocol.

\section{Results}

The individual moiety GFR values and the total GFR value of the affected kidneys obtained by in house software created at SSSIHL are shown in the Table1.

\begin{tabular}{|c|c|c|c|}
\hline Kidney & Upper moiety & Lower moiety & Total GFR \\
\hline LEFT & 21.36 & 6.88 & 28.24 \\
\hline LEFT & 22.23 & 22.97 & 45.20 \\
\hline LEFT & 37.66 & 16.72 & 54.38 \\
\hline LEFT & 17.62 & 6.67 & 24.29 \\
\hline LEFT & 33.11 & 33.68 & 66.79 \\
\hline LEFT & 31.1 & 15.4 & 46.5 \\
\hline LEFT & 39.26 & 13.35 & 52.61 \\
\hline LEFT & 36.56 & 15.73 & 52.29 \\
\hline LEFT & 26.77 & 33.56 & 60.33 \\
\hline LEFT & 18.08 & 2.02 & 20.1 \\
\hline LEFT & 7.98 & 11.85 & 19.83 \\
\hline LEFT & 9.03 & 15.63 & 24.66 \\
\hline RIGHT & 13.36 & 9.63 & 22.99 \\
\hline RIGHT & 7.81 & 4.05 & 11.86 \\
\hline RIGHT & 14.86 & 16.62 & 31.48 \\
\hline RIGHT & 16.01 & 10.18 & 26.19 \\
\hline RIGHT & 9.56 & 9.93 & 19.49 \\
\hline RIGHT & 44.46 & 25.01 & 69.47 \\
\hline RIGHT & 18.3 & 8.09 & 26.39 \\
\hline RIGHT & 32.1 & 12.01 & 44.11 \\
\hline
\end{tabular}

Table 1. Individual Moiety GFR value obtained by in house software created at SSSIHL

The scrutiny of the above table reveals that 15 out of 40 (37.5\%) moieties showed impaired functioning values. 10 out of $20(50 \%)$ lower moieties showed impaired functioning values and 5 out of 20 (25\%) of upper moieties had impaired functioning values. Indicating that in this study the lower moieties are predominantly affected when compared to the upper moieties. The total GFR value of the affected kidneys obtained by the in house software created at SSSIHL are tabulated along with the corresponding GFR value obtained by the ICON software and shown in the Table. 2 . 


\begin{tabular}{|c|c|c|}
\hline Affected kidney & Software Created at SSSIHL & Icon Software \\
\hline LEFT & 28.24 & 24 \\
\hline LEFT & 45.2 & 44 \\
\hline LEFT & 54.38 & 76 \\
\hline LEFT & 24.29 & 28 \\
\hline LEFT & 66.79 & 54 \\
\hline LEFT & 46.5 & 52 \\
\hline LEFT & 52.61 & 59 \\
\hline LEFT & 52.29 & 45 \\
\hline LEFT & 60.33 & 80 \\
\hline LEFT & 20.1 & 12 \\
\hline LEFT & 19.83 & 10 \\
\hline LEFT & 24.66 & 14 \\
\hline RIGHT & 22.99 & 24 \\
\hline RIGHT & 11.86 & 10 \\
\hline RIGHT & 31.48 & 23 \\
\hline RIGHT & 26.19 & 15 \\
\hline RIGHT & 19.49 & 70 \\
\hline RIGHT & 69.47 & 19 \\
\hline RIGHT & 26.39 & 58 \\
\hline RIGHT & 44.11 & \\
\hline
\end{tabular}

Table 2. GFR values of the affected kidneys obtained from ICON and MTNM Software of SSSIHL

The $\mathrm{T}$ test evaluation done in the online statistical calculator of Maths Portal org revealed that the mean values of the two groups showed no significant difference at $\mathrm{p}<0.05$. The calculated t value is smaller than the critical value $(0.0217<2.024)$, so the means are not significantly different. Pearson the $\mathrm{T}$ test evaluation done in the online statistical calculator of Maths Portal org revealed that the mean values of the two groups showed no significant difference at $\mathrm{p}<0.05$. The calculated $t$ value is smaller than the critical value $(0.0217<2.024)$, so the means are not significantly different. Pearson Correlation performed between the above two samples using statistical online calculators also showed the correlation coefficient of $r=0.9247$ and a mean difference of 0.09 indicating that the high correlation between them. The observed significance $\mathrm{p}$ value $<0.0001$ at $\mathrm{t}$ value of 10.27 , showed no significant difference detected between the two samples. This clearly proves that the in house software created at SSSIHL is producing identical results when compared with the widely used ICON software.

\section{Discussion}

The Glomerular Filtration Rate estimation using Tc99m DTPA by Gates method is versatile and unaffected by the delay in the acquisition and injection time has been documented by Guyangu Ma et al (8) recently proving the worthy nature of the Gates method. Similarly the utility of the same in various stages of the Chronic Diseases of the Kidney has been established by YungChun Ma et.al (9). However no attempts have been made so far to address the issue of estimation of individual moiety GFR in the case of congenital kidney anomaly of Double Moiety Kidneys in the literature. However the main limitation of this study is that it could analyse only 15 patients, which is a sub optimal number to derive any statistical validation. The gross variation between the combined values of the affected kidney by created software and the icon software is attributable to the non-identical drawing of the kidney outlines. This software should be tested on a wider platform to know the full utility and dependability of this software though the preliminary analysis is affirmative.

\section{Conclusion}

It can be concluded that the in house software created at SSSIHL based on the six compartmental modification of the Gates formula is quite useful in the evaluation of individual moieties of the kidneys in those patients with double moiety kidney either unilateral or bilateral affliction. This will help in the selection of Nephron sparing surgery in appropriate cases. Universal collaboration is solicited to evaluate this software for global utility.

\section{Conflicts of interest}

None.

\section{References}

1. Van Stekelen burg, Truijens, Van Vals, Kooman, A (1966) A quantitative theory of radioisotope renography with hippuran ${ }^{131} \mathrm{I}$. Phys Med Biol 11: 451-460.

2. Klopper JF, Hauser W, Atkins HL, Eckelman WC and Richards P. (1972) Evaluation of 99mTc- DTPA for the measurement of glomerular filtration rate. J Nucl Med 13: 107-110.

3. Houston, Sampson, Macleod (1979) A compartmental model for the distribution of $113 \mathrm{mIn}-\mathrm{DTPA}$ and $99 \mathrm{mTc}-(\mathrm{sn}) \mathrm{DTPA}$ in man following intravenous injection. Int J Nucl Med Biol. 6: 85-95.

4. Gary F. Gates (1982) Glomerular Filtration Rate: estimation from fractional renal accumulation of $99 \mathrm{mTc}$ DTPA (stannous). AmericalJournal of Roentgenology. 138(3):565-70

5. Gary F. Gates (1983) Split renal function testing using Tc-99m DTPA. A rapid technique for determining differential glomerular filtration. ClinNucl Med. Sep; 8(9):400-7.

6. Gary F. Gates (1984) Computation of glomerular filtration rate with Tc-99m DTPA: an in-house computer program. J Nucl Med. 25: 613-618

7. Matlab programming fundamentals, MATLAB, R2015b, Ccopy rights (1984) - 2015 by the MathWorks, Inc.

8. Guangyu Ma, Mingzhe Shao, Baixuan Xu, Jiahe Tian, and Yingmao Chen. (2019) Glomerular filtration rate measured by 99mTc-DTPA Gates method is not significantly affected by the premature or delayed initiation of image acquisition. Quant Imaging Med Surg. 9(6): 1103-1109.

9. Ying-Chun Ma, Li Zuo, Chun-Li Zhang, Mei Wang, Rong-Fu Wang and Hai-Yan Wang. (2007) Comparison of 99mTc-DTPA renal dynamic imaging with modified MDRD equation for 
glomerular filtration rate estimation in Chinese patients in different stages of chronic kidney disease. Nephrol Dial Transplant. 22: 417-423.

To Submit Your Article Click Here: Submit Manuscript

DOI: $10.31579 / 2690-8794 / 067$
Ready to submit your research? Choose Auctores and benefit from:

* fast, convenient online submission

* rigorous peer review by experienced research in your field

* rapid publication on acceptance

* authors retain copyrights

* unique DOI for all articles

* immediate, unrestricted online access

At Auctores, research is always in progress.

Learn more www.auctoresonline.org/journals/clinical-medical-reviewsand-reports 